\title{
An n-Dimensional Generalization of the Rhombus Tiling
}

\author{
Joakim Linde $^{1}$, Cristopher Moore ${ }^{2,3}$, and Mats G. Nordahl ${ }^{1,3}$ \\ ${ }^{1}$ Innovative Design, Chalmers University of Technology, Göteborg, Sweden \\ ${ }^{2}$ Computer Science Department and Department of Physics and Astronomy, University of New Mexico, Albuquerque \\ 87131 \\ ${ }^{3}$ Santa Fe Institute, 1399 Hyde Park Road, Santa Fe, NM 87501
}

Several classic tilings, including rhombuses and dominoes, possess height functions which allow us to 1) prove ergodicity and polynomial mixing times for Markov chains based on local moves, 2) use coupling from the past to sample perfectly random tilings, 3) map the statistics of random tilings at large scales to physical models of random surfaces, and and 4) are related to the "arctic circle" phenomenon. However, few examples are known for which this approach works in three or more dimensions. Here we show that the rhombus tiling can be generalized to $n$ dimensional tiles for any $n \geq 3$. For each $n$, we show that a certain local move is ergodic, and conjecture that it has a mixing time of $O\left(L^{n+2} \log L\right)$ on regions of size $L$. For $n=3$, the tiles are rhombohedra, and the local move consists of switching between two tilings of a rhombic dodecahedron. We use coupling from the past to sample random tilings of a large rhombic dodecahedron, and show that arctic regions exist in which the tiling is frozen into a fixed state. However, unlike the two-dimensional case in which the arctic region is an inscribed circle, here it seems to be octahedral. In addition, height fluctuations between the boundary of the region and the center appear to be constant rather than growing logarithmically. We conjecture that this is because the physics of the model is in a "smooth" phase where it is rigid at large scales, rather than a "rough" phase in which it is elastic.

Keywords: Tilings, Discrete Dynamical Systems, Quasicrystals

\section{Introduction}

Random tilings are a wonderful meeting place for computer scientists, physicists, and combinatorialists. They are easy to define and play with, but they raise deep issues about how discrete structures behave in the limit of large size, and how Monte Carlo algorithms can sample random combinatorial objects fairly and efficiently.

A number of classic two-dimensional tilings, including dominoes and rhombuses, possess a "height function" which, for a given configuration $a$, assigns an integer $h_{a}(v)$ to each vertex $v$ in the lattice. Configurations of the tiling then become surfaces in three-dimensional space. When it exists, this mapping helps us understand tilings in several ways:

1. Two tilings of the same simply-connected region correspond to two surfaces with the same boundary. We can then use the volume between them $\sum_{v}\left|h_{a}(v)-h_{b}(v)\right|$, or the Fourier transform of their difference, as a metric on the space of tilings. 
2. Local moves correspond to "flipping" a local maximum to a local minimum, and vice versa. Since a series of such flips can transform any tiling into any other, a Markov chain based on these flips is ergodic.

3. We can also define a partial order on tilings, where $a \succeq b$ if $h_{a}(v) \geq h_{b}(v)$ for all $v$. If the local move is monotone in this order, we can use coupling from the past to sample perfectly random tilings [41]. Specifically, if $\succeq$ forms a distributive lattice there are maximal and minimal tilings $\top$ and $\perp$, and we can bound the mixing time of a monotone Markov chain by bounding the time it takes $\top$ and $\perp$ to coalesce $[32,43,51]$.

4. In some cases, the statistics of random tilings map on to the statistics of elastic surfaces. Correlations between vertices in the lattice a distance $r$ apart then decay as power laws $r^{-\eta}$, where the exponent $\eta$ depends on the stiffness of the surface (see e.g. [6]).

5. Height functions can also give insight into the nature and interaction of topological defects, such as gaps in a tiling or places where a local constraint is violated [16, 33].

Height functions have also been applied by physicists to a variety of models, including ice models [2, 28], the triangular Ising antiferromagnet [5, 39], dimer models (as physicists call dominoes) [53, 30], and others.

As a generalization of the idea of an integer-valued height function, examples have been found where the height itself is a vector, giving an surface in four or more dimensions. These include antiferromagnetic Potts models, bond-coloring models, and fully-packed loop models on various lattices [19, 27, 42, 6, 34]. Height-like functions have also been defined which can take a dense set of values in the complex plane [35].

Another generalization is to have the height be an element of a non-Abelian group, in which case each type of tile corresponds to a relation in this group [9, 48, 22, 29, 46]. For dominoes, this representation reduces to the usual one where the heights are integers. In some cases, this group can be used to construct a linear-time algorithm to tell whether a given simply-connected region can be tiled [22]; this is interesting since this problem is NP-complete in general, even for some simple sets of tiles $[31,36]$ and undecidable for the infinite lattice [3, 47].

In general, these ideas seem to break down in three or more dimensions. Even simple tilings lack local moves which are ergodic, and we have very few tools for proving upper bounds on mixing times. Tiling folklore says that simple flips do not suffice for either $1 \times 1 \times 2$ dominoes or $2 \times 2 \times 1$ slabs, and this is illustrated in Figures 1 and 2. (Since domino tilings are perfect matchings of a bipartite graph there are other efficient methods for generating them randomly, but it would still be nice to understand them from a Markov chain point of view.)

The tilings introduced in this paper are similar in spirit to another generalization of the rhombus tiling, studied in $[23,14,12,11]$. These are two-dimensional tilings with $n$-dimensional height functions, while ours are $n$-dimensional tilings with 1-dimensional height functions. However, they are based on the same idea, namely projecting from an higher-dimensional cubic lattice onto a lower-dimensional space. This idea has been in the physics community for some time as a way to generate quasicrystals [40].

We know of very few other examples in three or more dimensions of models with a height function. One is the three-color model on the (hyper)cubic lattice, which we discuss in Section 6. Another is from recent work by Randall and Yngve [44]. They consider a set of triangular prisms designed so that successive 

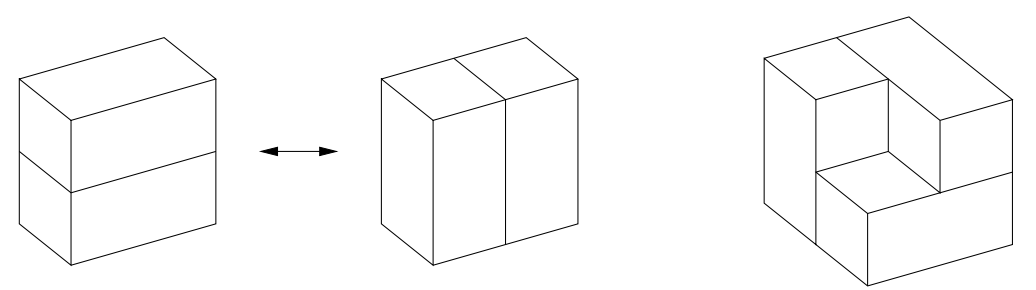

Fig. 1: The local flip on the left, and its rotations, are not ergodic for tilings of the cubic lattice by $1 \times 1 \times 2$ dominoes. The region shown on the right, while not simply-connected, can be linked to others like it with dominoes fitting into their missing corners to form a $2 \times 4 \times 4$ block where the local move cannot be applied anywhere.
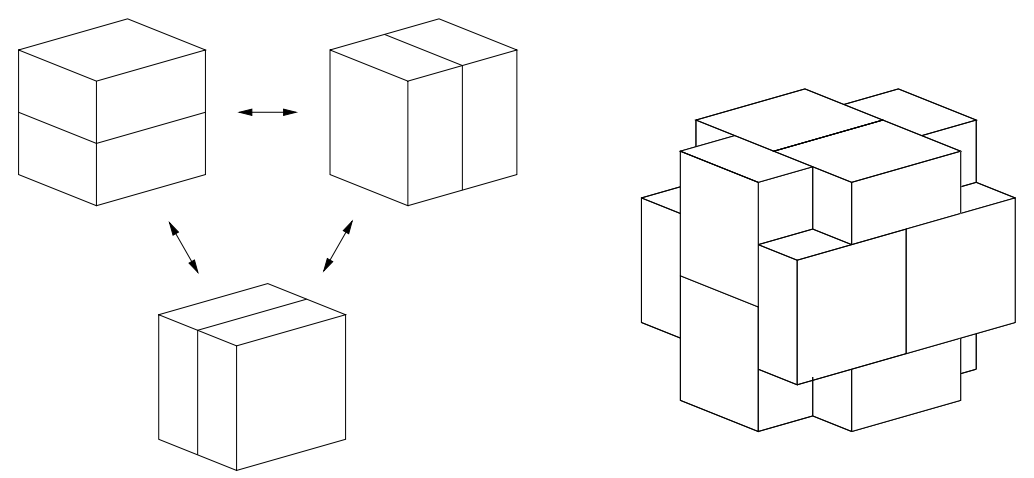

Fig. 2: The local flips on the left are not ergodic for tilings of the cubic lattice by $2 \times 2 \times 1$ slabs. The region shown on the right contains a cube which can be flipped, but the outer slabs cannot be changed into their mirror image. This region can easily be embedded in a $6 \times 6 \times 6$ cube. 

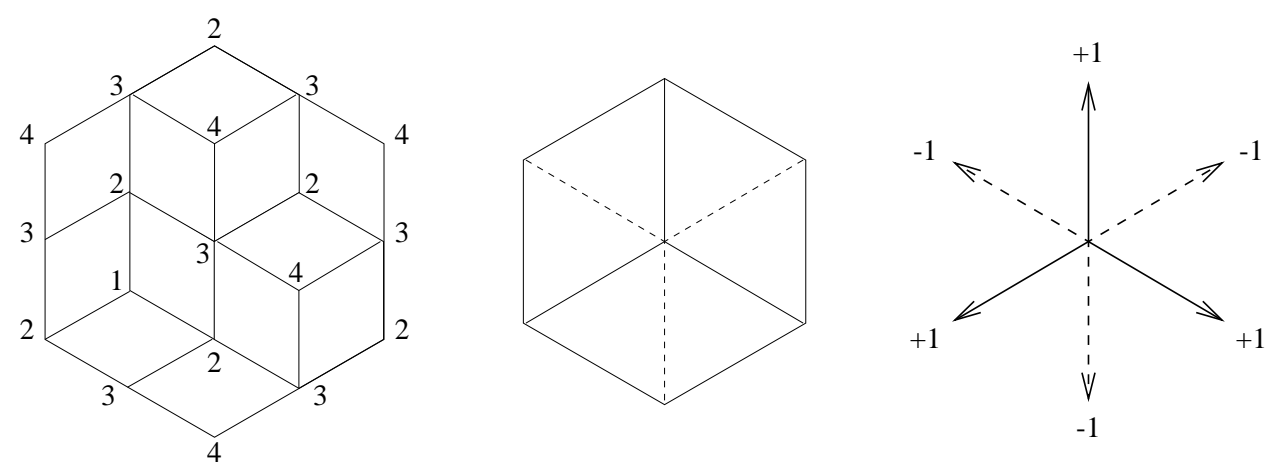

Fig. 3: The height function and the local move for the rhombus tiling.

layers correspond to domino tilings, increasing in the partial order from layer to layer. Thus their tiling bears the same relationship to domino tilings as rhombus tilings do to lattice paths. Our family of tilings is based on the same idea, and includes lattice paths and rhombus tilings as its 1- and 2-dimensional cases respectively.

We would like the reader to know that Dana Randall and Mihai Ciucu have thought a great deal about this model, and that Gary Yngve in fact performed some of the same experiments on coupling from the past that we have. While their work has not appeared in print, we are very grateful to Dana Randall for sharing it with us.

\section{The rhombus tiling and its $n$-dimensional generalization}

Rhombus tilings are one of the clearest illustrations of height functions, since they appear to the eye to be rooms partially filled with stacks of boxes. The local move or "flip," which rotates three rhombuses, is then equivalent to adding or removing a box. The height function is defined on the boxes' corners; if a corner's position in $\mathbb{Z}^{3}$ is $(x, y, z)$ then its height is $h=x+y+z$. Equivalently, moving along the tiles' edges increments or decrements the height depending on which of the six lattice directions we move along. All this is shown in Figure 3.

More formally, each configuration of the rhombus tiling is a projection along the diagonal $\mathbf{w}=(1,1,1)$ of a surface in $\mathbb{Z}^{3}$ composed of plaquets of the cubic lattice, such that any line parallel to $\mathbf{w}$ pierces the surface exactly once. The local move consists of shifting the surface up or down by adding or removing a cube, replacing its bottom faces with its top faces or vice versa. When projected, the square plaquets become rhombuses with angles $\pi / 6$ and $\pi / 3$, the vertices of the cubic lattice project onto those of the triangular lattice, and the height of a vertex corresponds to a point on the surface with position $\mathbf{x}$ is its component $\mathbf{x} \cdot \mathbf{w}$.

Given this, our generalization is entirely straightforward. A configuration of the $n$-dimensional tiling is a projection along $\mathbf{w}=(1,1, \ldots, 1) \in \mathbb{Z}^{n+1}$ of an $n$-dimensional surface composed of $n$-dimensional faces of the $(n+1)$-dimensional cubic lattice, such that any line parallel to $\mathbf{w}$ pierces the surface exactly once. The local move consists of shifting the surface by adding or removing an $(n+1)$-cube, replacing its bottom faces with its top faces or vice versa. When projected, these faces become rhombohedra, and the height of a vertex is the component along $\mathbf{w}$ of the corresponding point on the surface. 


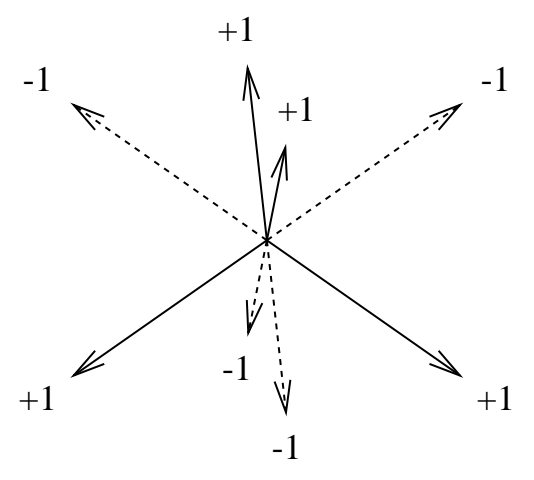

Fig. 4: The height function for the $n=3$ case. Moving along the edges of a tile increases or decreases the height depending on the direction in the body-centered cubic lattice, analogous to the height function for rhombuses shown in Figure 3.

For $n=3$, for instance, the 4-dimensional cubic lattice projects onto the body-centered cubic lattice, which has lattice directions $( \pm 1, \pm 1, \pm 1)$. Specifically, we can project the four basis vectors as follows:

$$
\begin{aligned}
& (1,0,0,0) \quad \mapsto \quad(+1,+1,+1) \\
& (0,1,0,0) \mapsto \quad(+1,-1,-1) \\
& (0,0,1,0) \mapsto \quad(-1,+1,-1) \\
& (0,0,0,1) \mapsto \quad(-1,-1,+1)
\end{aligned}
$$

Since the four basis vectors sum to $\mathbf{w}=(1,1,1,1)$, their projections sum to zero. Moving along the edge of a tile increases or decreases the height according to these lattice directions as shown in Figure 4.

Just as a cube projects to a hexagon in the 2-dimensional case, a 4-cube projects to rhombic dodecahedron; and just as a hexagon can be tiled with three rhombuses in two ways, corresponding to the top three and bottom three faces of a cube, a rhombic dodecahedron can be tiled with four rhombohedra in two ways, corresponding to the top four and bottom four cubic faces of a 4-cube. Switching between these two corresponds to adding or removing a 4-cube from the surface, and gives the local flip shown in Figure 5.

Formally, let $e_{i}=(0, \ldots, 1, \ldots, 0), 1 \leq i \leq n+1$, be the $i$ th basis vector of $\mathbb{Z}^{n+1}$, and let $v_{i}$ be $e_{i}$ projected along $\mathbf{w}$. We consider tilings of a region $U \subset \mathbb{R}^{n}$ by rhombohedral tiles, each of which has edges parallel to $n$ of the $v_{i}$. The vertices of the tiles fall on a lattice $V_{n}$ generated by the $v_{i}$, and for a given configuration $s$ of the $n$-dimensional tiling we write $h_{s}: V_{n} \rightarrow \mathbb{Z}$ for the height function, defined so that $h_{s}$ changes between vertices connected by an edge of a tile by \pm 1 depending on we are moving parallel or antiparallel to the appropriate $v_{i}$.

Then using standard reasoning, we can prove the following lemmas:

Lemma 1 Given a configuration s of the n-dimensional tiling on a simply connected region $U$, the height function $h_{s}$ as defined above is single-valued once the height of a single reference vertex $v_{0} \in V_{n}$ is chosen. 

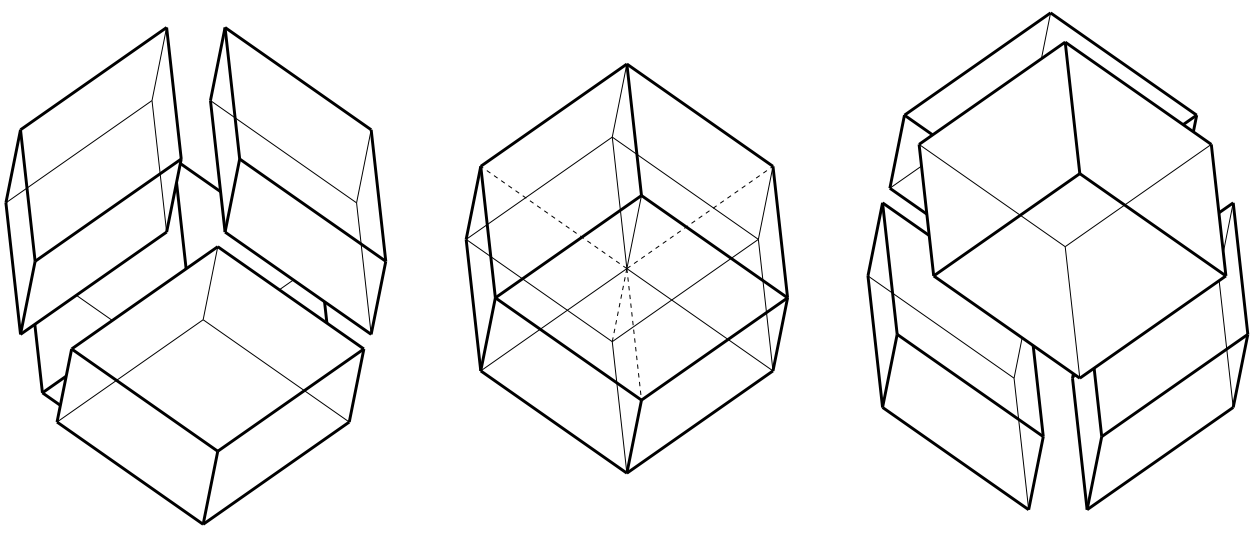

Fig. 5: The flip in the $n=3$ case. Just as a hexagon can be tiled by three rhombuses in two ways, corresponding to the top and bottom faces of a cube, a rhombic dodecahedron can be tiled by four rhombohedra in two ways, corresponding to the top and bottom faces of a 4-cube.

Proof. It suffices to show that the total change in height $\Delta h_{s}$ around any loop is zero; then every vertex $v$ has a height defined by traveling from $v_{0}$ to $v$, and $h_{s}(v)-h_{s}\left(v_{0}\right)$ is independent of the path taken. If $U$ is simply connected, any loop can written as an oriented union of elementary loops consisting of rhombic faces of the tiles. Since any such loop has the form $\pm v_{i}, \pm v_{j}, \mp v_{i}, \mp v_{j}$, the change in height around it is zero.

We note that if $U$ has non-contractible loops and is therefore not simply connected, $h_{s}$ can be multivalued with various "winding numbers" $\Delta h_{s}$ around these loops. The same thing happens in the presence of topological defects as discussed in Section 6.

Henceforth $U$ will denote a finite simply-connected region, and we write $T_{U}$ for the set of tilings of $U$. For $a, b \in T_{U}$ we fix $h_{a}\left(v_{0}\right)=h_{b}\left(v_{0}\right)=0$ at a reference vertex $v_{0}$ on $U$ 's boundary (in which case $h_{a}=h_{b}$ everywhere along the boundary) and define the partial order $a \succeq b$ if $h_{a}(v) \geq h_{b}(v)$ for all $v \in V_{n}$.

Since Lemma 1 shows every $a \in T_{U}$ actually corresponds to a surface in $\mathbb{R}^{n+1}$, we can also associate $a$ with the set $S_{a}$ of $(n+1)$-cubes "under" this surface, i.e. such that their positions' component along $\mathbf{w}$ is less than $h_{a}$, and their component transverse to $\mathbf{w}$ is inside $U$. Then $a \succeq b$ if and only if $S_{a} \supseteq S_{b}$.

Lemma 2 For a given simply-connected region $U$, the partial order $\succeq$ defines a distributive lattice on $T_{U}$.

Proof. Given $a, b \in T_{U}$, we can define tilings $a \vee b$ and $a \wedge b$ such that $S_{a \vee b}=S_{a} \cup S_{b}$ and $S_{a \wedge b}=S_{a} \cap S_{b}$, or equivalently $h_{a \vee b}(v)=\max \left(h_{a}(v), h_{b}(v)\right)$ and $h_{a \wedge b}(v)=\min \left(h_{a}(v), h_{b}(v)\right)$. Distributivity follows easily.

It follows that there are maximal and minimal tilings of $U, \top$ and $\perp$, such that $\top \succeq s \succeq \perp$ for all $s \in T_{U}$.

We now define and analyze a simple Markov chain $\mathcal{M}_{\text {flip }}$ based on our local moves:

Definition 1 Let $\mathcal{M}_{\text {flip }}$ be a Markov chain on $T_{U}$ defined as follows. At each step it chooses a vertex $v$ in the interior of $U$ and a direction $z= \pm 1$, both uniformly at random. If $z=+1$ (resp. $z=-1$ ) it attempts to increase (decrease) the height at $v$ by adding (removing) an $(n+1)$-cube to the surface corresponding to the current configuration. If the move cannot be applied at $v$ with direction $b, \mathcal{M}_{\mathrm{flip}}$ does nothing. 
Note that since the surface corresponding to the tiling must be transverse to $\mathbf{w}, \mathcal{M}_{\text {flip }}$ can only increase (decrease) the height at a vertex which is a local minimum (maximum) of the height.

We can show that $\mathcal{M}_{\text {flip }}$ is ergodic, i.e. that it can connect any tiling in $T_{U}$ to any other, and that it converges to the uniform distribution:

Lemma 3 For any simply-connected region $U$, $\mathcal{M}_{\text {flip }}$ converges to the uniform distribution on $T_{U}$.

Proof. It is trivial to see that $\mathcal{M}_{\text {flip }}$ is ergodic, since any two sets of $(n+1)$-cubes $S_{a}, S_{b}$ corresponding to tilings $a, b \in T_{U}$ can be transformed into each other by adding or removing $(n+1)$-cubes in the right places. Moreover, $\mathcal{M}_{\text {flip }}$ performs moves and their reverses with the same probability, so it satisfies detailed balance, and since it possesses self-loops it converges to the uniform distribution.

In order to use coupling from the past, we wish to show that $\mathcal{M}_{\text {flip }}$ is monotone: that is, if $a \succeq b$ and we try the same move in both $a$ and $b$ (i.e. we try to flip the same vertex in the same direction in both tilings) then the resulting tilings obey $a^{\prime} \succeq b^{\prime}$. This is easy to show:

Lemma 4 For any simply-connected region $U, \mathcal{M}_{\text {flip }}$ is monotone in $\succeq$.

Proof. Suppose $\mathcal{M}_{\text {flip }}$ attempts a move at a vertex $v$ in direction $z=+1$, and suppose $a \succeq b$ so that $h_{a}(v) \geq h_{b}(v)$. If $h_{a}(v)>h_{b}(v)$ this will remain true even if $\mathcal{M}_{\text {flip }}$ adds a cube to $S_{b}$ and not to $S_{a}$. If $h_{a}(v)=h_{b}(v)$ and $v$ is a local minimum of $h_{b}$, it must also be a local minimum of $h_{a}$, and $\mathcal{M}_{\text {flip }}$ will add a cube to both $S_{a}$ and $S_{b}$. In either case $h_{a^{\prime}}(v) \geq h_{b^{\prime}}(v)$. The case $z=-1$ is similar.

\section{The frozen region}

Armed with ergodicity and monotonicity, we can generate perfectly random tilings using coupling from the past. Specifically, we generated random tilings of rhombic dodecahedra of varying sizes. The maximal and minimal tilings $T$ and $\perp$ of this region correspond to a four-dimensional room full of 4-cubes and an empty room respectively. We apply the coupling to these two states, attempting the same moves of $\mathcal{M}_{\text {flip }}$ in each one. If after a certain amount of time $\top$ and $\perp$ have coalesced into a single state, since $\mathcal{M}_{\text {flip }}$ is monotone every initial state has coalesced, since they are squeezed between $\top$ and $\perp$. Since this means that $\mathcal{M}_{\text {flip }}$ has forgotten its initial state, we know that we have reached the uniform distribution. If they have not yet coalesced, we start farther back in time, doubling the number of moves, and so on, always ending with the same sequence of moves we tried before as described by Propp and Wilson [41].

In Figure 6 we show a random tiling of a rhombic dodecahedron of radius $L=64$. Just as in random rhombus tilings of a large hexagon or domino tilings of the Aztec diamond [7, 8, 20], there is a frozen region near the corners where with high probability the tiling is periodic, achieving the steepest possible gradient in the height, and an interior region where the entropy of the tiling resides. (In the figure the frozen regions are removed, showing only the interior.) Since these two-dimensional cases are frozen outside an "arctic circle," we might have hoped that this region would be an "arctic sphere," such as Randall and Yngve observe for tilings of an Aztec octahedron with triangular prisms. However, it appears to be octahedral, with flat triangular faces.

Note that the eight blunt corners of the rhombic dodecahedron (where three rhombuses meet at their obtuse corners) are the maxima and minima of the height function, while the six sharp corners (where four rhombuses meet at their acute corners) are saddle points. It is not surprising, then, that the frozen region does not form an inscribed sphere, since this would have frozen regions near both the sharp corners and the blunt ones; frozen regions are formed by maxima and minima of the height, not by saddle points. 
In this sense, the sharp corners are more like the midpoints of the two-dimensional hexagon than they are like its corners, so it makes sense that the interior region would touch them. However, this does not explain why there would be an "arctic octahedron" with flat triangular faces beneath the blunt corners. While we lack even a heuristic argument for the following conjecture, we make it anyway:

Conjecture 1 For $n \geq 3$ the frozen region in a random tiling of a projected $(n+1)$-cube has flat faces.

Note that this can also be taken as a conjecture about the shape of a typical boxed space partition in $n \geq 3$ dimensions, just as rhombus tilings correspond to boxed plane partitions [8]. We discuss this in Section 7.

\section{Mixing times}

Luby, Randall and Sinclair [32], Wilson [51], and Randall and Tetali [43] derive upper bounds on mixing times for rhombus tilings. In this section we sketch their results, discuss the difficulties of generalizing them to the $n$-dimensional case, and present a conjecture based on our numerical simulations. We denote the probability of an event as $\operatorname{Pr}[\cdot]$ and the expectation of a variable as $\mathrm{E}[\cdot]$.

Given probability distributions $P$ and $Q$ on $T_{U}$, their total variation distance is half the $L_{1}$ norm of their difference:

$$
\|P-Q\|=\frac{1}{2} \sum_{x \in T_{U}}|P(x)-Q(x)|
$$

Then if $P_{t}(x)$ is the probability distribution after $t$ steps of a Markov chain $\mathcal{M}$ with initial state $x$, then one definition of the distance from equilibrium is the total variation distance between distributions with different initial states:

$$
\bar{d}_{t}=\max _{x, y}\left\|P_{t}(x)-P_{t}(y)\right\|
$$

The $\varepsilon$-mixing time of $\mathcal{M}$ is then

$$
\tau(\varepsilon)=\max _{x, y} \min \left\{t: \bar{d}_{t} \leq \varepsilon \text { for all } t^{\prime} \geq t\right\}
$$

Now we consider a coupling $\mathcal{M}$, where starting from $x$ after $t$ steps we have a tiling $\mathcal{M}^{t}(x)$. Using a simple first-moment bound, and the fact that by monotonicity if $T$ and $\perp$ have coalesced than everything has, we have

$$
\bar{d}_{t} \leq \operatorname{Pr}\left[\mathcal{M}^{t}(\top) \neq \mathcal{M}^{t}(\perp)\right]
$$

Thus if with high probability $\top$ and $\perp$ have coalesced, we are close to the uniform distribution. More generally, if $T$ is the average time for $T$ and $\perp$ to coalesce, we have (e.g. [1])

$$
\tau(\varepsilon) \leq T e \log (1 / \varepsilon)
$$

In [32] we start by considering lattice paths, or one-dimensional random walks, and a Markov chain that flips local maxima and local minima. (This is in fact the one-dimensional case of $\mathcal{M}_{\text {flip. }}$.) The area between two paths is used as a metric, and we are interested in the distance $\Phi$ between the top and bottom paths. They show that $\mathrm{E}[\Delta \Phi] \leq 0$ and $\mathrm{E}\left[(\Delta \Phi)^{2}\right] \geq 1 / 2 L$, and then use a Martingale argument to show that $T=O\left(L^{6}\right)$. 


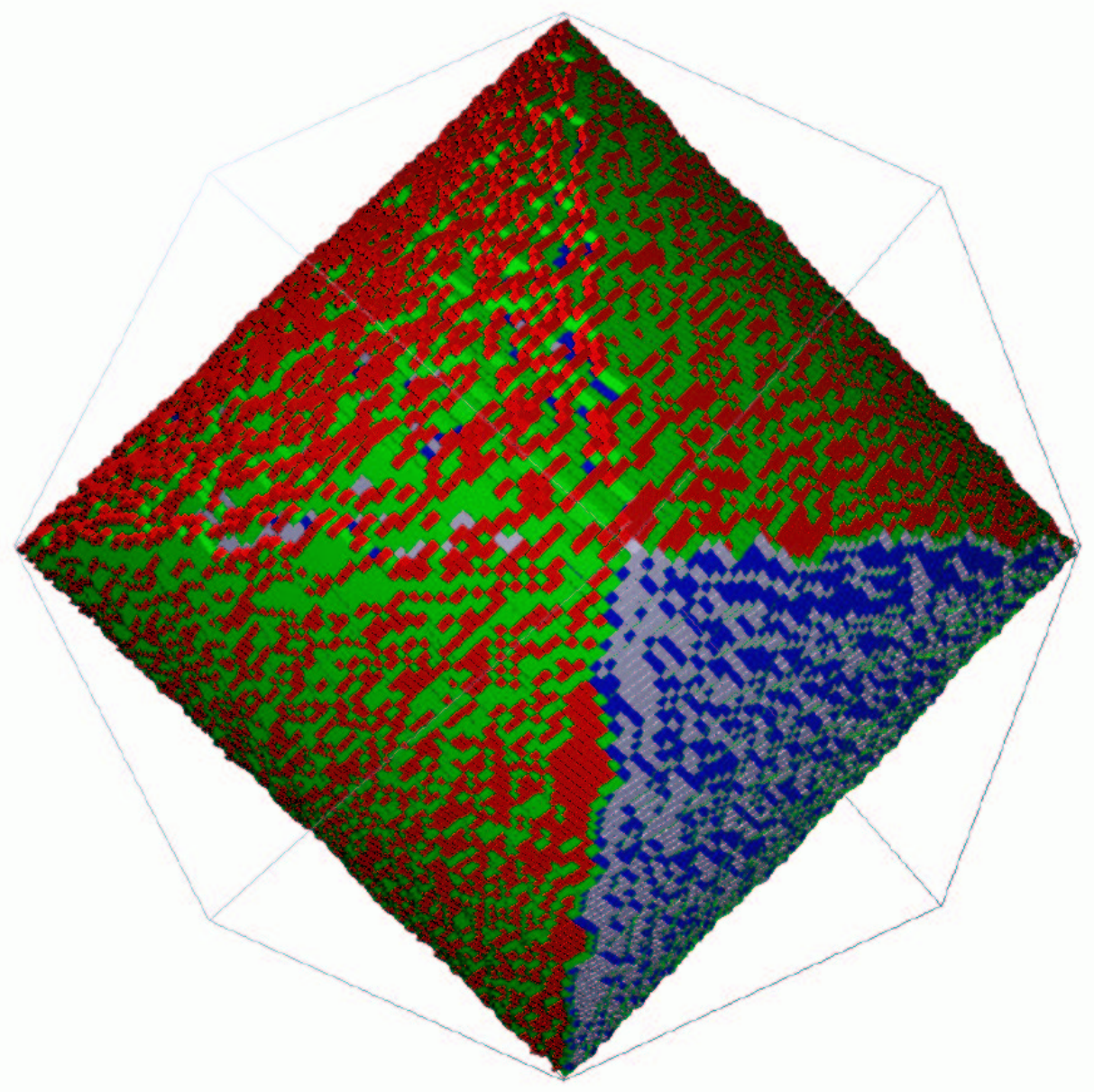

Fig. 6: A random tiling of a rhombic dodecahedron of radius $L=64$, formed by coupling from the past, with the frozen regions removed. The interior region appears to be octohedral, and touches the six sharp corners of the dodecahedron where the height function has a saddle point. 
In [51] this argument is improved, and made tight, by defining $\Phi$ to be a Fourier coefficient of the difference between two paths. We slightly simplify the argument of [51] by using $e^{i \beta x / L}$ instead of $\cos \beta x / L$ :

$$
\Phi_{\beta}=\sum_{x=0}^{L} e^{i \beta x / L}\left(h_{\top}(x)-h_{\perp}(x)\right)
$$

Now suppose that we choose a position $x$ to apply a flip to a path with height $h$. If $h(x+1)=h(x-1)=$ $h(x)+1$ then with probability $1 / 2$ we increase $h(x)$ by 2 , and with probability $1 / 2$ we leave it alone. If $h(x+1)=h(x)+1$ and $h(x-1)=h(x)-1$, then no move is possible. The other two cases are similar, and in all cases the expected height at $x$ after the move is the average of its neighbors' heights,

$$
\mathrm{E}\left[h^{\prime}(x)\right]=\frac{1}{2}(h(x-1)+h(x+1))
$$

Summing over all $x$ and ignoring the fact that we never flip the ends, this gives us

$$
\mathrm{E}\left[\Delta \Phi_{\beta}\right]=\frac{1}{L}\left(\frac{e^{i \beta / L}+e^{-i \beta / L}}{2}-1\right) \Phi_{\beta}=\frac{\cos \beta / L-1}{L} \Phi_{\beta}
$$

This is maximized when $\beta=\pi$, whereupon taking $L \rightarrow \infty$ gives

$$
\mathrm{E}\left[\Delta \Phi_{\pi}\right]=-\frac{\pi^{2}}{2 L^{3}} \Phi_{\pi}
$$

Thus, unlike the area between the paths, this definition of distance contracts reliably at each sstep of the coupling. If $\Phi_{0}$ is the initial distance between $\top$ and $\perp$, after $t$ steps

$$
\mathrm{E}\left[\left|\Phi_{\beta}\right|\right] \leq\left|\Phi_{0}\right| e^{-\frac{\pi^{2} t}{2 L^{3}}}
$$

for all $\beta$. Since $\Phi_{0} \leq \sum_{x} h_{\top}(x)-h_{\perp}(x) \leq L^{2}$, and since the smallest possible nonzero value of $|\Phi|$ is 1 , after a time

$$
t=\frac{2 L^{3}}{\pi^{2}} \log \frac{\Phi_{0}}{\varepsilon} \leq \frac{4 L^{3}}{\pi^{2}} \log \frac{L}{\varepsilon}
$$

the top and bottom paths have coalesced with probability $1-\varepsilon$, so

$$
\tau(\varepsilon)=O\left(L^{3} \log (L / \varepsilon)\right)
$$

In fact, this bound is tight, since at this $\tau$ times a smaller constant, $\left|\Phi_{\pi}\right|$ is bounded away from zero so that $\mathcal{M}^{t}(\top)$ and $\mathcal{M}^{t}(\perp)$ are still distinct with probability $1-o(1)$.

In two dimensions, we can regard rhombus tilings as families of lattice paths forming a increasing chain in the partial order. The difficulty is that adjacent paths can block each other from flipping. To get around this problem, Luby, Randall and Sinclair [32] consider a Markov chain $\mathcal{M}_{\mathrm{loz}}$ that includes tower moves where we add or remove a vertical stack of cubes all at once. With such moves, we can always flip a path up or down by also flipping those adjacent to it as shown in Figure 7.

By setting the probability of a tower move adding or removing $m$ cubes to $1 / m$, we can then restore the linearity of Equation 1. Since the probability that a particular location in a particular path is chosen for 


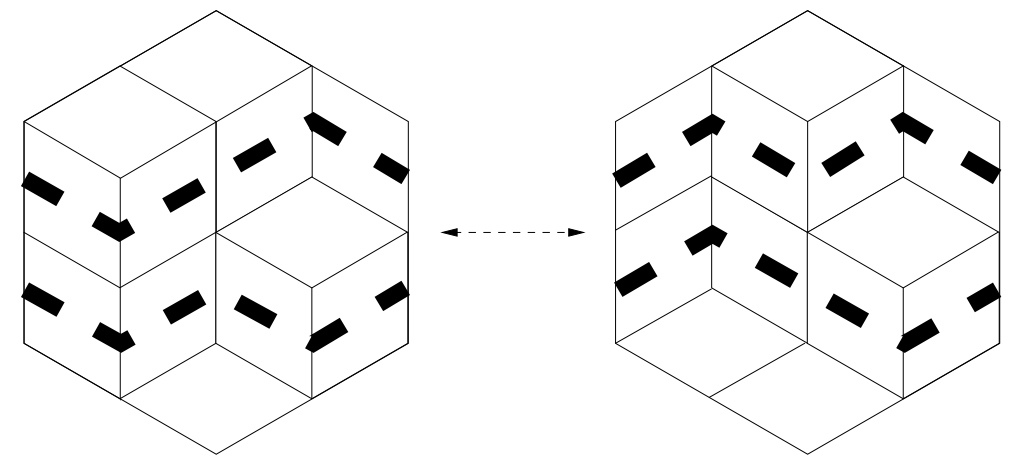

Fig. 7: The mapping from rhombus tilings to families of paths, and the effect of a tower move. We can flip the lower path up, but only by flipping the one above it as well. This corresponds to adding a stack of cubes.

a flip is inversely proportional to the area, $\mathrm{E}[\Delta \Phi]$ in Equation 2 becomes proportional to $1 / L^{2}$ instead of $1 / L$, giving us a mixing time of $\tau(\varepsilon)=O\left(L^{4} \log (L / \varepsilon)\right)$.

It is surprisingly difficult to show that this bound holds for the local Markov chain $\mathcal{M}_{\text {flip }}$ as well, even though this is borne out by numerical work of Cohn (unpublished), physical arguments of Henley on domino tilings and two-dimensional models with height functions in general [18], and numerical work by Destainville on a generalized lozenge tiling of the octagon [10]. The best known upper bound is by Randall and Tetali [43], who use the comparison method of Diaconis and Saloff-Coste [13] to bound how tower moves can be simulated by local moves. They are able to derive an upper bound of $O\left(L^{8} \log L\right)$ on the mixing time of $\mathcal{M}_{\text {flip }}$.

Note that lattice paths and rhombus tilings are the one- and two-dimensional cases of our tiling, and rhombus tilings correspond to chains of lattice paths. We can generalize this as follows:

Lemma 5 A configuration of the $n$-dimensional tiling corresponds to a chain of $(n-1)$-dimensional tilings, increasing in the partial order.

Proof. Note that the paths in Figure 7 consist of those rhombuses where one of the edges is vertical, and are separated by tiles whose edges are along the other two directions. In the same way, in a configuration of the $n$-dimensional tiling, the tiles with an edge parallel to $v_{n+1}$ (say) form layers, separated by tiles with edges parallel to $v_{1}, \ldots, v_{n}$. The surface of each layer, projected along $v_{n+1}$, forms a configuration of the $(n-1)$-dimensional tiling. Successive layers are increasing in the partial order since the intervening tiles, if any, are topologically $n$-cubes, and adding these corresponds to upward flips of the $(n-1)$-dimensional tiling.

Applying this inductively, we see that the $n$-dimensional tiling corresponds to an $(n-1)$-dimensional lattice of one-dimensional tilings, i.e. lattice paths. As in the two-dimensional case, a path can be blocked from flipping by adjacent paths. We would like to define a Markov chain with multi-dimensional tower moves that gives each location of each path a chance of flipping inversely proportional to the volume, in which case $\mathrm{E}[\Delta \Phi]$ in Equation 2 would be proportional to $1 / L^{n}$ and we would have a mixing time of $O\left(L^{n+2} \log L\right)$. Unfortunately, in more than two dimensions the tower moves we need appear to be irreversible [45]. 


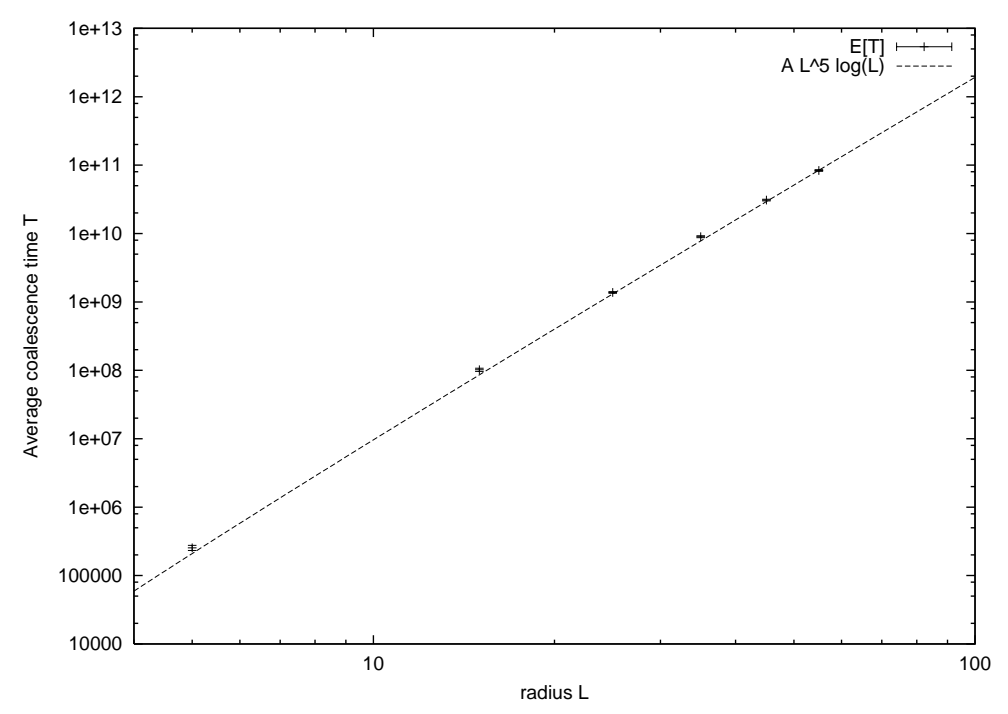

Fig. 8: A log-log plot of the coalescence time for rhombic dodecahedra of radius $L$. Each point is the average of 20 trials. The data is closely fit by the form $A L^{5} \log L$, providing support for Conjecture 2 .

As mentioned above, even in the two-dimensional case we currently lack a proof that local moves have the same mixing time as tower moves, $O\left(L^{4} \log L\right)$. However, assuming that some form of the argument of [51] could go through, we make the following conjecture:

Conjecture 2 The $\varepsilon$-mixing time of $\mathcal{M}_{\text {flip }}$ for the $n$-dimensional tiling on regions of size $L$ is $\tau(\varepsilon)=$ $O\left(L^{d+2} \log (L / \varepsilon)\right)$.

In Figure 8, we provide numerical evidence for this conjecture in the three-dimensional case. We graph the average coalescence time $T(L)$ over 20 trials each for rhombic dodecahedra of radius $L=5,15,25,35,45$, and 55. The data is closely fit by the form $T(L)=A L^{5} \log L$.

\section{Height fluctuations: rough vs. smooth}

In this section we discuss the connection between tilings with height functions and the statistical physics of an elastic surface. Note to the reader: this section and the next are non-rigorous. However, Kenyon has recently obtained rigorous results for domino and lozenge tilings [24, 25].

Given a height function, a random tiling corresponds to a random surface. If the tiling is physically in a "rough" phase, we expect this surface to act like an elastic sheet with some stiffness constant $K$. It then has an effective free energy proportional to the integral of the gradient of the height squared,

$$
G=\frac{1}{2} K \int \mathrm{d} \mathbf{x}|\nabla h|^{2}
$$

If we Fourier transform the height by writing

$$
\tilde{h}(\mathbf{q})=\int \mathrm{d} \mathbf{x} e^{i \mathbf{q} \cdot \mathbf{x}} h(\mathbf{x})
$$


then the free energy decouples into a sum of independent quadratic terms,

$$
G=\frac{1}{2} K \int \mathrm{d} \mathbf{q}|\mathbf{q}|^{2} \tilde{h}(\mathbf{q})
$$

This form for the free energy is called a Gaussian effective field. It gives a power spectrum for height fluctuations decreasing quadratically with $|\mathbf{q}|$,

$$
\mathrm{E}\left[|\tilde{h}(\mathbf{q})|^{2}\right]=\frac{1}{K|\mathbf{q}|^{2}}
$$

In real space, this gives spatial correlations between the heights at points a distance $r$ apart of

$$
\mathrm{E}\left[(h(0)-h(\mathbf{r}))^{2}\right]=C(\mathbf{r}) \simeq \frac{1}{\pi K} \log r+C
$$

for large $r$, where $C$ is a constant. Kenyon has shown that domino tilings are in fact governed by a Gaussian field [24], and that height fluctuations in domino and lozenge tilings do in fact have this form [25].

Correlations in local variables - the probability that a vertex is the top left corner of a vertical domino, say - then decay as power laws $r^{-\eta}$, where $\eta$ depends on the stiffness $K$ and the frequency of the local variable as a function of $h$. All this generalizes to higher-dimensional height functions as well, where $K$ becomes a tensor rather than a scalar [6,34].

On the other hand, if the tiling is in a "smooth" phase, then the heights of two vertices a distance $r$ apart remain tightly correlated even as $r \rightarrow \infty$, so that $h(0)-h(\mathbf{r})$ stays constant. In Figure 9, we measure the height fluctuations for random tilings of rhombic dodecahdra of varius radii, specifically the height change from their center to their boundary. Since the height change appears not to increase with size, we make the following conjecture:

Conjecture 3 For $n \geq 3$, the physics of our tiling is in a smooth phase.

We note that various marginal behaviors can occur at or near the transition from rough to smooth [6,37], where the height fluctuations increase with size but more slowly than in Equation 3.

\section{Topological defects}

In addition to perfect tilings, we can also consider tilings with defects, i.e. gaps between tiles. We might define a Markov chain which starts with a random configuration containing many defects, and then tries to relax it towards a perfect tiling by making local moves which minimize an appropriate energy function. Physically, this corresponds to starting with an infinite-temperature configuration and then cooling it towards absolute zero. From the computer science point of view, understanding this relaxation process could be important if we don't know how to generate an initial perfect tiling as the starting point for a Markov chain.

When they exist, height functions can give some physical insight into these defects and their interactions. Specifically, if an $n$-dimensional model has a height function, we expect it to have $(n-2)$ dimensional topological defects, where the height function changes by an amount $q=\Delta h$ (and so becomes multivalued) when we travel in a loop around the defect. Physicists refer to $q$ as the topological charge or, for dislocations in lattices, the Burgers vector. For $n=2$ these defects are points, and for $n=3$ they form so-called vortex loops, where $\Delta h$ is nonzero around any closed path linked with a vortex loop. 


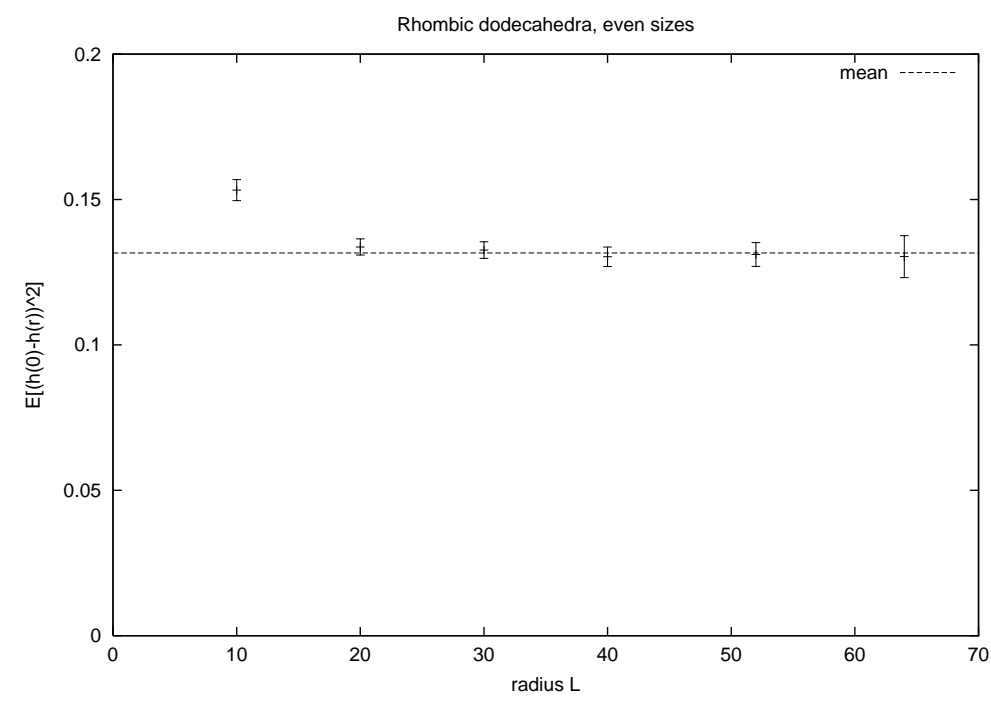

Fig. 9: Measurements of height fluctuations in our model, specifically the height change from the center of a rhombic dodecahedron of a given size to its boundary. If our tiling were in a rough phase, $(h(0)-h(r))^{2}$ would increase logarithmically with $r$. Instead, it seems to be roughly constant, suggesting that it is in a smooth, or rigid, phase.

For instance, in the three-color model of the square lattice (or as physicists say, the three-state antiferromagnetic Potts model at zero temperature), if we call the colors 0,1 , and 2 , we can define a height function such that the color at a vertex $v$ is $s(v)=h(v) \bmod 3$, and such that $\Delta h= \pm 1$ between neighbors [26]. The total height change is then zero around a square plaquet. However, if there is a defect consisting of a pair of neighbors with the same color as shown in Figure 10, its charge is $q=\Delta h= \pm 6$. (Note that this model is equivalent to Eulerian orientations of the square lattice, or as physicists say, the six-vertex ice model [38].) Similarly, in the domino tiling, the charge of a "hole" is +4 and -4 for a black hole and a white hole respectively (where we have two-colored the lattice as a checkerboard), and in the rhombus tiling the charge of a triangular hole is +3 or -3 depending on whether the hole points left or right in Figure 3.

The height function for the three-color model generalizes to the $n$-dimensional cubic lattice, since for
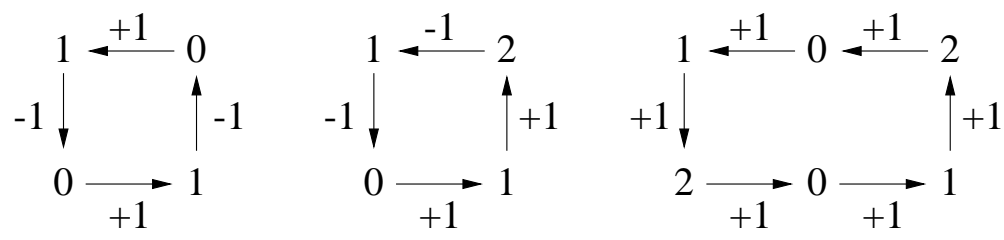

Fig. 10: The height function for the three-color model on the square lattice. The height is defined so that the color is $s=h \bmod 3$, and so that $\Delta h= \pm 1$ between neighbors. In the absence of defects, $\Delta h=0$ around a square plaquet and so $h$ is single-valued, but around a defect we have a topological charge of $\Delta h= \pm 6$. 
all $n$ we have square plaquets with $\Delta h=0$. In three dimensions its defects form vortex loops, as shown in Figure 11. Here we are partway through a relaxation process where we start with a random assignment of colors to the cells of the cubic lattice, and then perform local moves which minimize the number of like neighbors. While we have not yet studied defects in our rhombohedral tilings, we expect them to form vortex loops in $n=3$, membranes in $n=4$, and so on.

Using a Gaussian effective field theory as in Section 5, we expect a height gradient a distance $r$ away from such a defect of $q / 2 \pi r$, and a free energy between two defects with charges $q, q^{\prime}$ a distance $r$ apart proportional to

$$
G \sim \iint \mathrm{d} x \mathrm{~d} y|\nabla h|^{2} \sim q q^{\prime} \log r
$$

In a local Markov chain, this free energy induces a force between defects of

$$
F=-\nabla G \sim \frac{q q^{\prime}}{r}
$$

so that defects of opposite charge are attracted, and those of like charge are repelled. Note that this force is purely entropic: it happens simply because the number of configurations of the rest of the lattice increases as two defects of opposite type are brought together. Specifically, the effective free energy here is a constant minus the entropy, which is the log of the number of tilings with a given arrangement of defects.

Fisher and Stephenson [16] showed that the number of domino tilings with one hole of each type does in fact behave in this way as a function of the distance between them. Moore et al. [33] measured these forces numerically in the three-color model on the square lattice, and Zeng et al. [52] performed a similar experiment for a fully-packed loop model. In all three cases, defects of opposite charge a distance $r$ apart are indeed attracted to each other with a force proportional to $1 / r$.

Such a force is similar to the Biot-Savart law of electromagnetism [4], where $\nabla h$ plays the role of the magnetic field. In two dimensions defects act like vortices or current elements piercing the plane, and in three dimensions vortex loops act like current loops. We know of no experiments on entropic forces in three or more dimensions. However, if we are right that in $n \geq 3$ our tilings are in a smooth phase, the effective Gaussian field theory will not apply.

Higher-dimensional models which lack a height function can have other types of defects. For instance, three-dimensional domino tilings and Eulerian orientations of the cubic lattice have point defects rather than vortex loops - black and white holes for dominoes, and sources and sinks for Eulerian orientations. Such defects act like magnetic monopoles, where the charge is now the divergence of a vector field (in the case of Eulerian orientations, of the orientations themselves). In a Gaussian effective field theory, these would have electrostatic forces between them proportional to $1 / r^{n-1}$. The nature of defects in a given model presumably depends on some sort of discrete homotopy class, but how this works remains an important open question in higher-dimensional tilings.

\section{Conclusions and open questions}

In addition to Conjectures 1-3 above, we propose a number of open questions.

1. Perfect matchings? Random tilings first arose in physics in the statistical mechanics of dimers on a lattice. Domino and rhombus tilings correspond to dimer coverings, i.e. perfect matchings, on the square and hexagonal lattices respectively. This allows us to express the number of tilings as a 


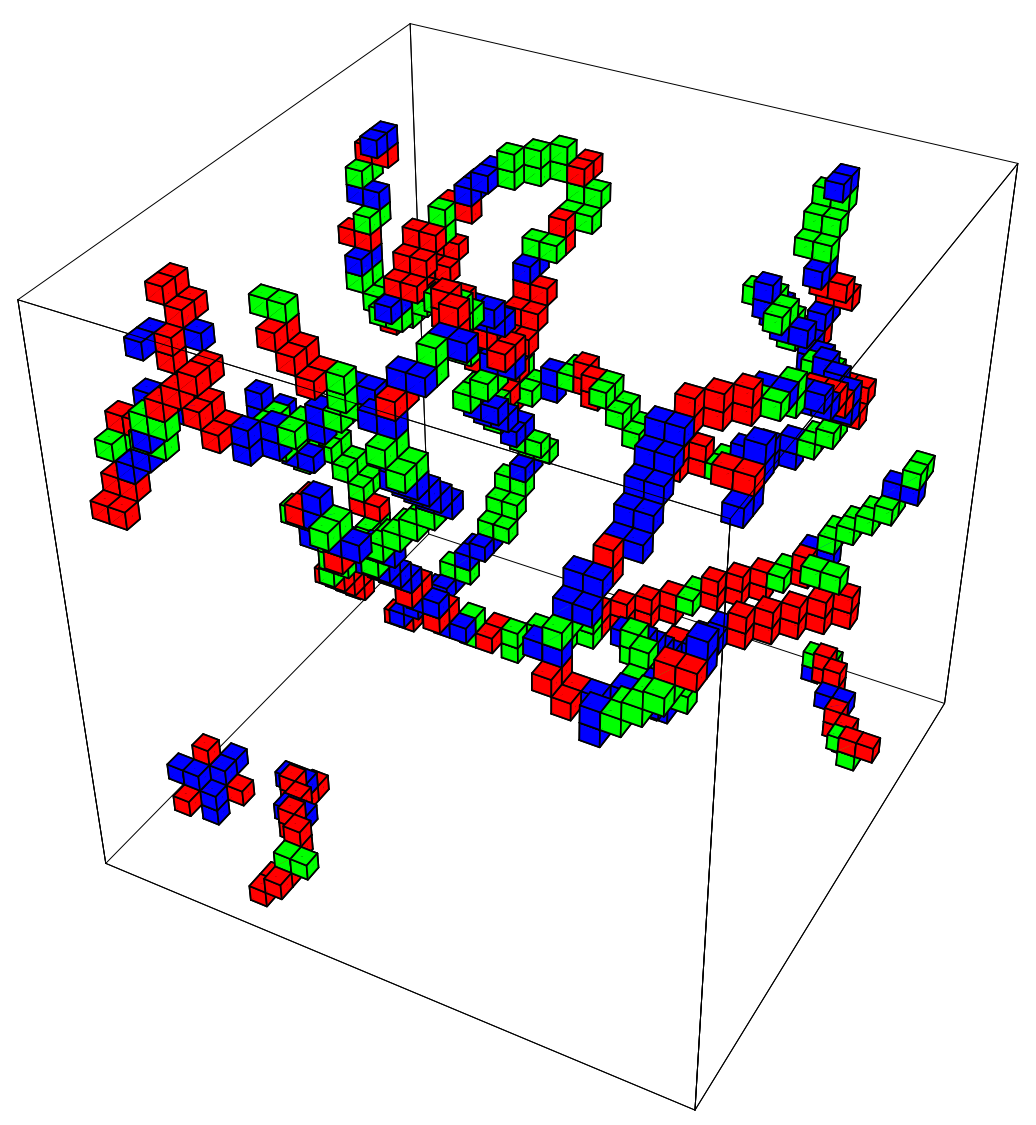

Fig. 11: Vortex loops in the three-coloring model of a $32 \times 32 \times 32$ cubic lattice with toroidal boundary conditions. Starting from a random assignment of colors in which many pairs of neighbors have the same color, we perform a Markov chain which minimizes the number of like neighbors. Partway through this relaxation process there are vortex loops composed of like pairs, around which the height function changes by \pm 6 . Only these pairs are shown. 
permanent, which on these lattices can be written as a Pfaffian determinant $[15,17,21]$. It would be nice if this mapping generalized to the $n$-dimensional case; however, we have not been able to connect the three-dimensional tiling studied here to a perfect matching on any lattice.

2. Antiferromagnets? Rhombus tilings are also equivalent to the triangular Ising antiferromagnet at zero temperature. It would be nice to know if $n$-dimensional rhombohedral tilings correspond to simple statistical models, such as antiferromagnets or antiferromagnetic Potts models (colorings) on an appropriate lattice.

3. Space partitions? There is a simple mapping between rhombus tilings of a hexagon and boxed plane partitions, i.e. functions $f$ from rectangles to non-negative integers such that $f$ is nondecreasing across any row or column. These correspond to solid Young diagrams, or more prosaically piles of cubes, and $f$ bears a simple relationship to the height function. The arctic circle phenomenon then tells us about the shape of a typical boxed plane partition [8]. In the same way, our conjecture about the frozen region in $n \geq 3$ corresponds to a conjecture about typical boxed space partitions, which might be resolved using other combinatoric arguments.

4. Cluster algorithms? A number of models, including 3-colorings of the square lattice [49], 4colorings of the triangular lattice [34], and domino and rhombus tilings and perfect matchings in general [50, pp. 74-75], have ergodic Markov chains where each move changes an entire loop or cluster on the lattice rather than a single vertex. These cluster moves are popular among physicists because they seem to mix much more quickly, although this has not been proved. In the $n$-dimensional case, we might imagine cluster moves which update a loop of towers, a tower of loops, or an $(n-1)$-dimensional closed surface.

Acknowledgements. We are grateful to Michel Morvan for a visit to the Santa Fe Institute, at which these tilings came up as a geometrical instantiation of zonotope tilings; to Dana Randall for extensive conversations and for sharing unpublished work; and to David Wilson for a reading of the manuscript. C.M. is supported partly by Sandia National Laboratory's SURP program, and thanks Molly Rose and Spootie the Cat for their support.

\section{References}

[1] D. Aldous, "Random walks on finite groups and rapidly mixing Markov chains." Séminaire de Probabilités XVII 1981/82, Springer Lecture Notes in Mathematics 986 243-297.

[2] H. van Beijeren, "Exactly solvable model for the roughening transition of a crystal surface." Phys. Rev. Lett. 38 (1977) 993-996.

[3] R. Berger, "The undecidability of the domino problem." Memoirs Amer. Math. Soc. 66 (1966) 1-72.

[4] See for example

http://hyperphysics.phy-astr.gsu.edu/hbase/magnetic/biosav.html

[5] H.W.J. Blöte and H.J. Hilhorst, "Roughening transitions and the zero-temperature triangular Ising antiferromagnet." J. Phys. A 15 (1982) L631-L637. 
[6] J.K. Burton, Jr. and C.L. Henley, "A constrained Potts antiferromagnet model with an interface representation." J. Phys. A 30 (1997) 8385-8413.

[7] H. Cohn, R. Kenyon, and J. Propp, "A variational principle for domino tilings." J. Amer. Math. Soc. 14 (2001) 297-346.

[8] H. Cohn, M. Larsen, and J. Propp, "The shape of a typical boxed plane partition." New York J. Math. 4 (1998) 137-165.

[9] J.H. Conway and J.C. Lagarias, "Tilings with polyominoes and combinatorial group theory." J. Combin. Theory Ser. A $\mathbf{5 3}$ (1990) 183-206.

[10] N. Destainville, "Flip dynamics in octagonal rhombus tiling sets." Preprint cond-mat/0101413.

[11] N. Destainville, R. Mosseri, and F. Bailly, "Fixed-boundary octagonal random tilings: a combinatorial approach.” J. Stat. Phys. 102(1/2) (2001) 147-190.

[12] N. Destainville, R. Mosseri, and F. Bailly, "Configurational entropy of codimension-one tilings and directed membranes." J. Stat. Phys. 87 (1997) 697-.

[13] P. Diaconis and L. Saloff-Coste, "Comparison theorems for reversible Markov chains." Ann. Appl. Prob. 3(3) (1993) 696-730.

[14] S. Elnitsky, "Rhombic tilings of polygons and classes of reduced words in Coxeter groups." J. Combin. Theory 77 (1997) 193-221.

[15] M.E. Fisher, "Statistical mechanics of dimers on a plane lattice." Phys. Rev. 124 (1961) 1664-1672.

[16] M.E. Fisher and J. Stephenson, "Statistical mechanics of dimers on a plane lattice II: Dimer correlations and monomers." Phys. Rev. 132 (1963) 1411-1431.

[17] H.N.V. Temperley and M.E. Fisher, "Dimer problem in statistical mechanics-an exact result." Phil. Mag. 6 (1961) 1061-1063.

[18] C. Henley, "Relaxation time for a dimer covering with height representation." J. Stat. Phys. 89(3/4) (1997) 483-507.

[19] D.A. Huse and A.D. Rutenberg, "Classical antiferromagnets on the Kagomé lattice." Phys. Rev. $B$ 45 (1992) 7536-7539.

[20] W. Jockusch, J. Propp, and P. Shor, "Random domino tilings and the arctic circle theorem." Preprint math. CO/9801068.

[21] P.W. Kasteleyn, "The statistics of dimers on a lattice, I: the number of dimer arrangements on a quadratic lattice." Physica 27 (1961) 1209-1225.

[22] C. Kenyon and R. Kenyon, "Tiling a polygon with rectangles." Proc. 33rd Symp. Foundations of Computer Science (1992) 610-619.

[23] R. Kenyon, "Tilings of polygons with parallelograms." Algorithmica 9 (1993) 382-397. 
[24] R. Kenyon, "Dominos and the Gaussian free field.” Preprint math-ph/0002027.

[25] R. Kenyon, “Local statistics of lattice dimers.” Preprint math . CO/0105054.

[26] J. Kolafa, "Monte Carlo study of the three-state square Potts antiferromagnet." J. Phys. A: Math. Gen. 17 (1984) L777-L781.

[27] J. Kondev and C.L. Henley, "Four-coloring model on the square lattice: A critical ground state." Phys. Rev. B 52 (1995) 6628-6639.

[28] H.J.F. Knops, "Renormalization connection between the eight-vertex model and the Gaussian model.” Ann. Phys. 128 (1981) 448-462.

[29] J.C. Lagarias and D.S. Romano, "A polynomial tiling problem of Thurston and its configurational entropy.” J. Combin. Theory Ser. A 63 (1993) 338-358.

[30] L.S. Levitov, "Equivalence of the dimer resonating-valence-bond problem to the quantum roughening problem.” Phys. Rev. Lett. 64 (1990) 92-94.

[31] H. Lewis, "Complexity of solvable cases of the decision problem for predicate calculus." Proc. 19th Symp. on Foundations of Computer Science (1978) 35-47.

[32] M. Luby, D. Randall, and A. Sinclair, "Markov chain algorithms for planar lattice structures.” Proc. 36th Symposium on Foundations of Computer Science (1995) 150-159.

[33] C. Moore, M.G. Nordahl, N. Minar, and C. Shalizi, "Vortex dynamics and entropic forces in antiferromagnets and antiferromagnetic Potts models." Physical Review E 60 (1999) 5344-5351.

[34] C. Moore and M.E.J. Newman, "Height representation, critical exponents, and ergodicity in the four-state triangular Potts antiferromagnet.” Journal of Statistical Physics 99 (2000) 661-690.

[35] C. Moore and I. Pak, "Ribbon tile invariants from signed area." To appear in Journal of Combinatorial Theory Ser. A.

[36] C. Moore and J.M. Robson, "Hard tiling problems with simple tiles." Submitted to Discrete and Computational Geometry.

[37] C. Moore, "Height representation and long-range order in random trimer tilings of the square lattice." In preparation.

[38] M.E.J. Newman and G.T. Barkema, Monte Carlo Methods in Statistical Physics. Oxford University Press, Oxford (1999).

[39] B. Nienhuis, H.J. Hilhorst, and H.W.J. Blöte, “Triangular SOS models and cubic-crystal shapes.” J. Phys. A 17 (1984) 3559-3581.

[40] R. Penrose, Bull. Inst. Math. and its Appl. 10 (1974) 266-.

[41] J. Propp and D. Wilson, "Exact Sampling with Coupled Markov Chains and Applications to Statistical Mechanics." Random Structures and Algorithms 9 (1996) 223-252. 
[42] R. Raghavan, C.L. Henley, and S.L. Arouh, "New two-color dimer models with critical ground states." J. Stat. Phys. 86 (1997) 517-550.

[43] D. Randall and P. Tetali, "Analyzing Glauber dynamics by comparison of Markov chains.” Journal of Mathematical Physics 41 (2000) 1598-1615.

[44] D. Randall and G. Yngve, "Random three-dimensional tilings of Aztec octahedra and tetrahedra: an extension of domino tilings." Proc. 11th Symp. on Discrete Algorithms (2000).

[45] D. Randall, personal communication.

[46] E. Rémila, "Tiling groups: new applications in the triangular lattice." Discrete and Combinatorial Geometry 20 (1998) 189-204.

[47] R.M. Robinson, "Undecidability and nonperiodicity of tilings of the plane." Inventiones Math. 12 (1971) 177-.

[48] W.P. Thurston, “Conway's tiling groups.” Am. Math. Monthly 97 (1990) 757-773.

[49] J.-S. Wang, R.H. Swendsen, and R. Kotecký, "Antiferromagnetic Potts models," Phys. Rev. Lett. 63 (1989) 109-112, and "Three-state antiferromagnetic Potts models: A Monte Carlo study," Phys. Rev. B 42 (1990) 2465-2474.

[50] D. Wilson, Exact Sampling with Markov Chains. Ph.D. thesis, MIT Mathematics Department, 1996.

[51] D. Wilson, "Mixing times of lozenge tiling and card shuffling Markov chains." Preprint math.PR/0102193.

[52] C. Zeng and P.L. Leath, "Application of polynomial algorithms to a random elastic medium." cond-mat/9810154, and C. Zeng, P.L. Leath, and D.S. Fisher, "Absence of two-dimensional Bragg glasses." cond-mat/9807281.

[53] W. Zheng and S. Sachdev, "Sine-Gordon theory of the non-Néel phase of two-dimensional quantum antiferromagnets." Phys. Rev. B 40 (1989) 2704-2707. 